\title{
Effect of Raloxifene Treatment on Osteocyte Apoptosis in Postmenopausal Women
}

\author{
Huib W. van Essen · Paulien J. Holzmann • \\ Marinus A. Blankenstein · Paul Lips • \\ Nathalie Bravenboer
}

Received: 12 March 2007 / Accepted: 11 June 2007/Published online: 4 August 2007

(C) Springer Science+Business Media, LLC 2007

\begin{abstract}
Increased osteocyte apoptosis, as the result of estrogen deficiency, could play a role in the decrease of bone mass and bone strength seen in postmenopausal osteoporosis. We investigated whether treatment with raloxifene of postmenopausal women with osteoporosis affects osteocyte apoptosis. Transiliac bone biopsies were obtained from 26 osteoporotic women at baseline and after 2 years of treatment with placebo or raloxifene. Immunohistochemical detection of cleaved caspase-3 was performed on sections from nondecalcified bone biopsies to visualize apoptosis. In the trabecular bone total osteocytes, positively stained osteocytes and empty lacunae were counted and percent positive cells and percent empty lacunae determined. Statistical evaluation was performed by Wilcoxon's paired t-test and Spearman's rank correlations. There was no significant difference in percentage positive osteocytes between baseline and follow-up biopsies in both the placebo and the raloxifene groups. The percentage empty lacunae increased significantly in the placebo group $(11.20 \pm 1.43$ vs. $9.00 \pm 2.25, P=0.014)$ but not in the raloxifene group. At baseline in both groups combined, there was a negative correlation between indices of bone remodeling and the percentage positive osteocytes (bone formation rate/bone volume $r=-0.67, P=0.001$ ). We found no direct evidence for an effect of raloxifene treatment on osteocyte apoptosis, but small effects of
\end{abstract}

H. W. van Essen $(\bowtie) \cdot$ P. J. Holzmann · P. Lips Department of Endocrinology, VU University Medical Center, 1007 MB Amsterdam, The Netherlands

e-mail: hw.vanessen@vumc.nl

M. A. Blankenstein · N. Bravenboer

Department of Clinical Chemistry, VU University Medical

Center, 1007 MB Amsterdam, The Netherlands raloxifene treatment cannot be excluded. The percent of apoptotic osteocytes was dependent on the level of bone remodeling in an individual.

Keywords Apoptosis · Histomorphometry · Osteocyte · Postmenopausal osteoporosis · Raloxifene

Osteocytes play a key role in the maintenance of bone mass and structure. The main function of osteocytes is to sense mechanical stress in the bone [1]. Osteocytes respond to this with the production of nitric oxide, prostaglandins, and other factors which are believed to restrain osteoclastic bone resorption or promote bone formation [2, 3]. A putative second role of osteocytes is to direct bone remodeling to foci of microdamage. Osteocyte apoptosis around the site of microdamage attracts bone remodeling cells, which resorb the damaged bone and replace it with new mechanically competent bone $[4,5]$.

Postmenopausal osteoporosis generally results in a decrease in bone mineral density (BMD) and a higher susceptibility for osteoporotic fractures. It is characterized by high bone remodeling, with bone resorption exceeding bone formation. Estrogen deficiency results in an increase in the recruitment and activity of both osteoblasts and osteoclasts. It also leads to increased osteoclast survival, while the life span of the osteoblast is decreased [6, 7]. Recently it has been shown both in humans and in rats that estrogen deficiency also leads to increased osteocyte apoptosis [8, 9]. The resulting decrease in osteocyte number could, in time, impair the response of bone to mechanical stress and lead to an accumulation of microdamage. Therefore, it would be of interest to know whether therapies aimed at reducing postmenopausal bone loss affect the survival of osteocytes. 
Raloxifene is a selective estrogen receptor modulator (SERM) that can bind to the estrogen receptors ER $\alpha$ and $\mathrm{ER} \beta$. It has been shown to increase BMD and reduce vertebral fracture risk in women with postmenopausal osteoporosis [10, 11]. In vitro studies suggest that raloxifene exerts its effect, like estrogen, through modulation of the number and activity of both osteoclasts and osteoblasts [12]. Kousteni et al. [13] have recently shown, using in vitro experiments, that raloxifene does not inhibit etoposide-induced apoptosis of rat calvarial osteoblasts; but no clinical studies have examined the effect of raloxifene on osteocyte apoptosis in postmenopausal women.

In this study, we investigated whether treatment of postmenopausal women with raloxifene for 2 years would change osteocyte survival in trabecular bone and whether the level of osteocyte apoptosis would be associated with the level of bone remodeling.

\section{Materials and Methods}

\section{Study Outline}

All women in the study were participants in the Multiple Outcomes of Raloxifene Evaluation (MORE) trial. This was a placebo-controlled, double-blind, multicenter trial to test the efficacy of raloxifene. Details of this study have already been published elsewhere [10]. Briefly, 7,705 women were at least 2 years postmenopausal and had osteoporosis as defined by a BMD of at least 2.5 standard deviations (SDs) below the young adult mean and/or one or more vertebral fractures. They were randomly assigned to one of the following three treatment groups: placebo, 60 $\mathrm{mg}$ /day raloxifene, and $120 \mathrm{mg} /$ day raloxifene. Additionally, all women received daily vitamin D (400-600 IU) and calcium (500 mg). Among the exclusion criteria for this study were the use of androgen, calcitonin, or bisphosphonates within the previous 6 months; oral estrogen within the previous 2 months; fluoride therapy for more than 3 months during the previous 2 years; or systemic glucocorticoid therapy for more than 1 month within the past year. During the study the women received no therapy with respect to their osteoporosis other than the study drugs. The use of other prescription drugs and over-the-counter drugs such as sedatives, antibiotics, and paracetamol was equally distributed in the placebo and raloxifene groups.

In this study, bone biopsies were obtained at baseline and after 2 years from 26 women who were enrolled in the European centers of the MORE trial. These women were part of the bone histomorphometry substudy of the MORE trial that included 65 women from two centers in the United States and two centers in Europe. Bone biopsies from the other 39 women were not available for sectioning.
All women had given their informed consent, and the study was approved by the institutional ethics review boards.

Markers of bone turnover that were measured were serum osteocalcin, bone-specific alkaline phosphatase (BSAP), and urinary type 1 collagen C-telopeptide corrected for creatinine (CTX-I). Data are from the baseline and 24-month evaluations.

\section{Bone Biopsies}

The women received two doses of tetracycline with a 12day interval. Transverse biopsy specimens were taken from the anterior iliac crest, the 2-year biopsy being on the opposite side from the baseline biopsy. The bone biopsies were immediately fixed in cold $4 \%$ phosphate-buffered formaldehyde, dehydrated in graded ethanol, and embedded in methylmethacrylate (MMA; BDH Chemicals, Poole, England) supplemented with $20 \%$ plastoid-N (Röhm und Haas, Darmstadt, Germany), $2.0 \mathrm{~g} / \mathrm{L}$ benzoylperoxide (Merck, Darmstadt, Germany), and N,N-dimethylaniline (Merck) [14]. Sections of $5 \mu \mathrm{m}$ were cut with a Jung (Nussloch, Germany) K Polycut microtome. Sections were stained with Goldner's trichrome method. Static and dynamic histomorphometric measurements were performed as previously reported $[15,16]$. Histomorphometric indices used for this study included the percentage of bone surface covered by osteoid (osteoid surface, OS/BS) and the amount of mineralized bone formed per year on a given bone area (bone formation rate/bone volume, BFR/BV) as bone formation indices and the percentage of bone surface covered by osteoclasts or appearing eroded (eroded surface, ES/BS) and the number of osteoclasts per bone area (osteoclast number, Ocl.N/B.Ar) as bone resorption indices $[17,18]$. Histomorphometric data and immunohistochemical data were obtained from the same biopsies.

\section{Immunohistochemistry}

Apoptotic cells were visualized by immunohistochemical detection of activated caspase-3. The antibody against cleaved caspase-3 specifically stains apoptotic cells. Unlike the terminal deoxynucleotidyl transferase-mediated deoxyuridine triphosphate nick end labeling (TUNEL) method, there is no staining of necrotic cells or cells with DNA damage. For optimal accuracy of the method, immunohistochemistry was performed on four $5 \mu \mathrm{m}$ sections of each biopsy, which were obtained with an interval of $30 \mu \mathrm{m}$. Sections were cut and transferred to poly-L-lysine-coated slides. After deplastification and rehydration, sections were decalcified for 10 minutes with $1 \%$ acetic acid. Antigen retrieval was performed by 30 - 
minute incubation with $0.5 \%$ saponin (Sigma, St. Louis, MO) in phosphate-buffered saline (PBS) and 10-minute incubation with $3.5 \mu \mathrm{g} / \mathrm{mL}$ DNAse II (Sigma) in $25 \mathrm{mM}$ Tris $+10 \mathrm{mM} \mathrm{MgSO}_{4}$. Sections were incubated with $3 \%$ $\mathrm{H}_{2} \mathrm{O}_{2}$ in methanol to block endogenous peroxidase and with $5 \%$ normal goat serum in PBS $+0.05 \%$ Tween to block nonspecific binding sites. Incubation with primary antibody was performed overnight at $4^{\circ} \mathrm{C}$ with $1 / 100$ rabbit-anticleaved caspase-3 antibody (Cell Signaling Technology, Beverly, MA) in PBS $+0.05 \%$ Tween. Sections were then incubated for 1 hour with $1 / 100$ biotin-labeled goat-anti-rabbit immunoglobulin G (Vector Labs, Burlingame, CA) in PBS $+0.05 \%$ Tween. The sections were incubated for 30 minutes with the $\mathrm{ABC}$ kit (Vector Labs) and developed for 10 minutes with 3,3'diaminobenzidine with nickel enhancement. Sections were counterstained with $0.025 \%$ toluidine blue in $\mathrm{H}_{2} \mathrm{O}$, dehydrated, and sealed in DEPEX mounting medium (BDH). Random quality-control sections were measured in each assay. Sections of human ileum were tested as a positive control for apoptotic cells.

\section{Cell Counting}

The identity of the sections was blinded, and they were randomly numbered. In each section, the total trabecular bone area was measured using Osteomeasure software (Osteometrics, Atlanta, GA). In the entire trabecular bone area, the total number of osteocytes, the number of cleaved caspase-3-positive osteocytes, and the number of empty lacunae were counted with x200 magnification. Artefacts, such as areas where bone marrow obscured the trabeculae or where trabeculae were crossed, were avoided. All sections were analyzed by the same investigator.

From these data we calculated the following parameters:

- percentage of positive osteocytes per total osteocytes (Pos.Ot.N/Tt.Ot.N)

- percentage of empty lacunae per total lacunae (EL.N/ Tt.L.N)

- empty lacunae per bone area (EL.N/B.Ar)

- total lacunae per bone area (Tt.L.N/B.Ar)

Calculations from the four sections per biopsy were averaged. The variation in the method was assessed by calculating the average standard deviation from all the quadruplicates according to the following formula:

$$
\sqrt{\frac{\sum_{j=1}^{n} \sum_{i=1}^{k}\left(X_{i j}-\overline{x_{j}}\right)^{2}}{\sum_{j=1}^{n}\left(n_{j}-1\right)}}
$$

in which $n$ is number of subjects, $k$ is number of measurements per subject, $n_{j}$ is number of measurements in subject $j, X_{i j}$ is score measurement $i$ in subject $j$, and $\overline{x_{j}}$ is mean score in subject $j$.

\section{Statistics}

All values are expressed as mean $\pm \mathrm{SD}$. Differences in the calculated parameters between the 2-year biopsies and the baseline biopsies within groups were tested using a nonparametric paired $t$-test (Wilcoxon signed rank test). Differences between the treatment groups in the percent change after 2 years of treatment were tested with a nonparametric $t$-test (Mann-Whitney signed rank test). Correlations between the calculated parameters and histomorphometric indices or biochemical markers were assessed with Spearman's rank correlations. Statistical analysis was performed using SPSS (Chicago, IL) 12.0 software.

\section{Results}

The data presented here are derived from 26 patients who attended the European centers of the MORE trial. Therefore, this study covers only part of the group of patients on which histomorphometry data were published earlier [16]. Of the 26 women in this study, 11 received placebo, ten received $60 \mathrm{mg} /$ day raloxifene, and five received $120 \mathrm{mg} /$ day raloxifene. Because the number of women in the 120 $\mathrm{mg} /$ day raloxifene group was small and no difference in results between the two raloxifene treatment groups was detected, as tested using the Mann-Whitney signed rank test, data from the two raloxifene groups were combined for further analysis. The average age of the women in the placebo group was $67.9 \pm 6.1$ years; in the raloxifene group the average age was $67.1 \pm 6.7$ years.

Staining for cleaved caspase-3 clearly identified apoptotic osteocytes (Fig. 1a); in the negative control no cells showed any staining. Figure $1 b$ shows an example of an empty lacuna. In the human ileum sections, clear positive staining of intestinal epithelial cells was seen at the luminal surface of the villi while the negative control showed no staining (Fig. 1c, d). Table 1 shows the baseline and followup results of the calculated parameters in the placebo group and the raloxifene group and the average standard deviation of the parameters. Direct comparison of the follow-up biopsy with the baseline biopsy revealed no difference in the percentage of positive osteocytes (Pos.Ot.N/Tl.Ot.N) in either the placebo group or the raloxifene group.

As empty lacunae are the result of osteocyte apoptosis and are only removed by bone remodeling, changes in the 
Fig. 1 Immunohistochemical staining of bone biopsy sections and human ileum sections for cleaved caspase-3. Human bone biopsy sections stained with cleaved caspase-3 (a, b). Arrows indicate apoptotic osteocyte (a) or empty lacuna (b). Human ileum sections stained with cleaved caspase-3 (c) or no first antibody (d). Arrows indicate apoptotic cells
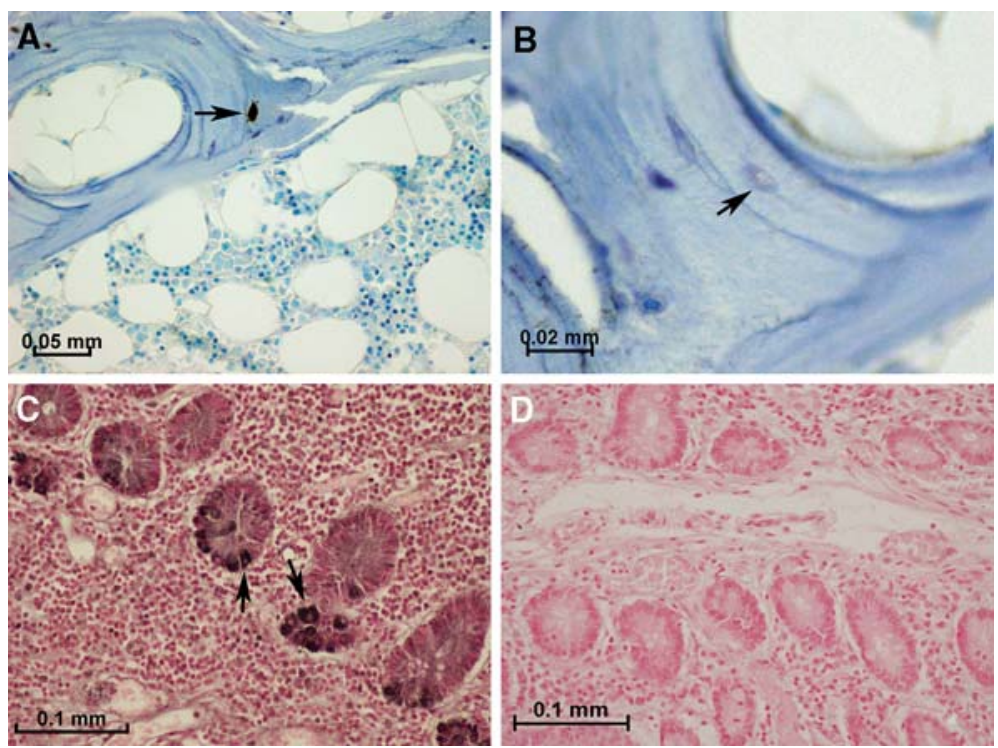

Table 1 Results of baseline and follow-up biopsies for the calculated parameters

\begin{tabular}{llll}
\hline Parameter (average standard deviation) & a & Time point & Placebo $(n=11, \text { mean } \pm \text { SD })^{\text {Raloxifene }^{\mathrm{b}}(n=15, \text { mean } \pm \text { SD })}$ \\
\hline Pos.Ot.N/Tt.Ot.N (\%) (1.28) & Baseline & $6.66 \pm 5.28$ & $5.30 \pm 3.51$ \\
& Follow-up & $6.82 \pm 5.27$ & $4.99 \pm 2.29$ \\
EL.N/Tt.L.N (\%) (2.43) & Baseline & $9.00 \pm 2.25$ & $9.11 \pm 2.88$ \\
& Follow-up & $11.20 \pm 1.43^{\mathrm{c}}$ & $9.74 \pm 1.91$ \\
EL.N/B.Ar (n/mm ${ }^{2}$ (6.29) & Baseline & $21.2 \pm 6.13$ & $21.0 \pm 6.49$ \\
& Follow-up & $24.5 \pm 3.68$ & $22.5 \pm 4.67$ \\
Tt.L.N/B.Ar. $\left(\mathrm{n} / \mathrm{mm}^{2}\right)(22.34)$ & Baseline & $233.9 \pm 28.2$ & $231.5 \pm 22.9$ \\
& Follow-up & $219.5 \pm 27.3$ & $231.1 \pm 19.6$
\end{tabular}

a Average standard deviation calculated from all quadruplicates

b The $60 \mathrm{mg} /$ day and $120 \mathrm{mg} /$ day raloxifene groups were combined

c Statistically significant difference $(P=0.014)$ from baseline value

number of empty lacunae could be indicative of changes in osteocyte apoptosis. In the placebo group, the percentage of empty lacunae (EL.N/Tt.L.N) increased significantly after 2 years (also shown in Fig. 2). The empty lacunar density (EL.N/B.Ar) showed a parallel change, but this was not significant. In the raloxifene group, the percentage of empty lacunae and the empty lacunar density did not increase significantly. The average standard deviation, calculated from the quadruplicate analysis, showed that the variation in the method was comparable to the variation between individuals, except for the measurement of percent positive cells, where the variation between the individuals was much higher.

Associations of bone remodeling parameters with osteocyte apoptosis and empty lacunae are shown in Table 2. At baseline, in the placebo group and the raloxifene group combined, there was a negative correlation of the histomorphometric indices (BFR/BV, OS/BS, ES/BS, and Ocl.N/ B.Ar) with the percent positive osteocytes (Pos.Ot.N/
Tt.Ot.N). Histomorphometric indices were not correlated with empty lacunae. The regression lines for BFR/BV with percent apoptotic osteocytes and percent empty lacunae are shown in Figure 3. Correlations between biochemical indices (BSAP, osteocalcin, and CTX-1) and percent apoptotic osteocytes or percent empty lacunae were not found.

No changes in the histomorphometric indices between baseline and follow-up were found, nor were there any differences between the placebo and raloxifene groups. All three biochemical markers showed a significant decrease at follow-up compared to baseline in the raloxifene group but not in the placebo group (Table 3).

\section{Discussion}

We investigated the effect of treatment with raloxifene for 2 years of postmenopausal osteoporotic women on osteocyte apoptosis as measured by activated caspase- 3 

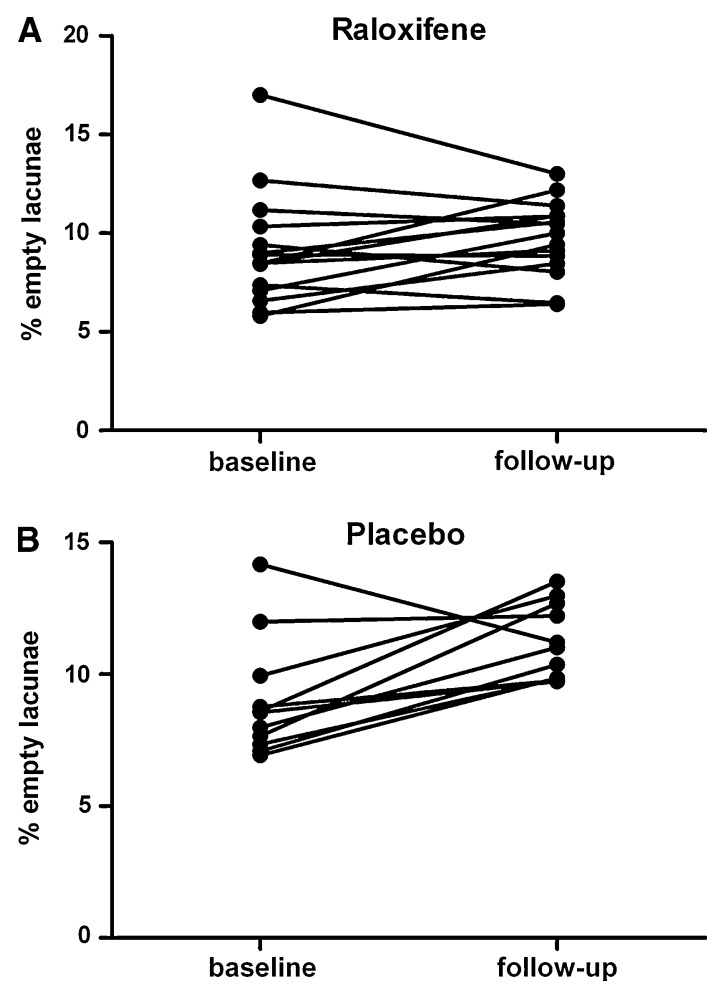

Fig. 2 Change in percentage empty lacunae after treatment for 2 years with either placebo (A) or raloxifene (B). Empty lacunae and total lacunae were counted in each biopsy. The increase in the placebo group was significant $(P=0.014)$

immunohistochemistry in iliac crest bone biopsies. Direct comparison of follow-up with baseline in the placebo and raloxifene groups did not show differences in the percent positive osteocytes. This suggests that raloxifene has little or no influence on osteocyte apoptosis. We did find a significant increase in the percentage of empty lacunae at 2 years in the placebo group, while there was no change in the raloxifene group. This lack of accumulation of empty lacunae in the raloxifene group could be the consequence of an inhibitory effect of raloxifene on osteocyte apoptosis. At baseline, histomorphometric indices of bone remodeling were inversely correlated with apoptotic osteocytes but not with empty lacunae. Biochemical markers of bone remodeling were not correlated with apoptotic osteocytes or empty lacunae.

This is the first study to investigate the effect of antiresorptive treatment on osteocyte apoptosis in human bone biopsies. So far, the effect of raloxifene on osteoblast or osteocyte apoptosis has only been studied in in vitro studies. Kousteni et al. [13] studied the effect of raloxifene on etoposide-induced apoptosis of rat calvarial osteoblasts and did not find protection against apoptosis. On the other hand, Olivier et al. [19] found that raloxifene protected the osteoblast-like cell line MC3T3-E1 against apoptosis induced by a high concentration of nitric oxide. Comparable clinical studies with hormone replacement therapy or bisphosphonates have not been published, but in vitro studies $[20,21]$ and studies with mice $[22,23]$ have shown that both these treatments have an inhibiting effect on osteocyte apoptosis induced by glucocorticoids or etoposide.

The difference between the raloxifene group and the placebo group in percent empty lacunae provides some evidence for an inhibitory effect of raloxifene on osteocyte apoptosis, although this effect was not reflected in the empty lacunar density. The fact that apoptotic osteocytes and empty lacunae change differently in response to raloxifene treatment could be explained by the comparatively short time that apoptosis can be detected. It has been shown that nonviable osteocytes are detectable for up to 16 weeks

Table 2 Association of histomorphometric indices and biochemical indices of bone remodeling with osteocyte apoptosis (Pos.Ot.N/Tt.Ot.N) and empty lacunae (E.L.N/Tt.L.N)

\begin{tabular}{|c|c|c|c|c|}
\hline & \multicolumn{4}{|c|}{ Histomorphometric indices } \\
\hline & OS/BS & $\mathrm{BFR} / \mathrm{BV}$ & ES/BS & Ocl.N/B.Ar \\
\hline \multirow[t]{2}{*}{$\%$ Positive osteocytes (Pos.Ot.N/Tt.Ot.N) } & $r=-0.64$ & $r=-0.67$ & $r=-0.48$ & $r=-0.43$ \\
\hline & $P=0.0004^{\mathrm{a}}$ & $P=0.001^{\mathrm{a}}$ & $P=0.01^{\mathrm{a}}$ & $P=0.03^{\mathrm{a}}$ \\
\hline \multirow[t]{4}{*}{ \% Empty lacunae (E.L.N/Tt.L.N) } & $r=0.03$ & $r=0.10$ & $r=0.05$ & $r=0.48$ \\
\hline & $P=0.87$ & $P=0.67$ & $P=0.80$ & $P=0.02^{\mathrm{a}}$ \\
\hline & \multicolumn{4}{|c|}{ Biochemical indices } \\
\hline & \multicolumn{2}{|c|}{ Osteocalcin } & BSAP & CTX \\
\hline \multirow[t]{2}{*}{$\%$ Positive osteocytes (Pos.Ot.N/Tt.Ot.N) } & \multicolumn{2}{|c|}{$r=-0.35$} & $r=-0.43$ & $r=-0.40$ \\
\hline & \multicolumn{2}{|c|}{$P=0.13$} & $P=0.05$ & $P=0.09$ \\
\hline \multirow[t]{2}{*}{ \% Empty lacunae (E.L.N/Tt.L.N) } & \multicolumn{2}{|c|}{$r=0.10$} & $r=0.13$ & $r=0.31$ \\
\hline & \multicolumn{2}{|c|}{$P=0.66$} & $P=0.56$ & $P=0.19$ \\
\hline
\end{tabular}

${ }^{a}$ Statistically significant $(P<0.05)$ 

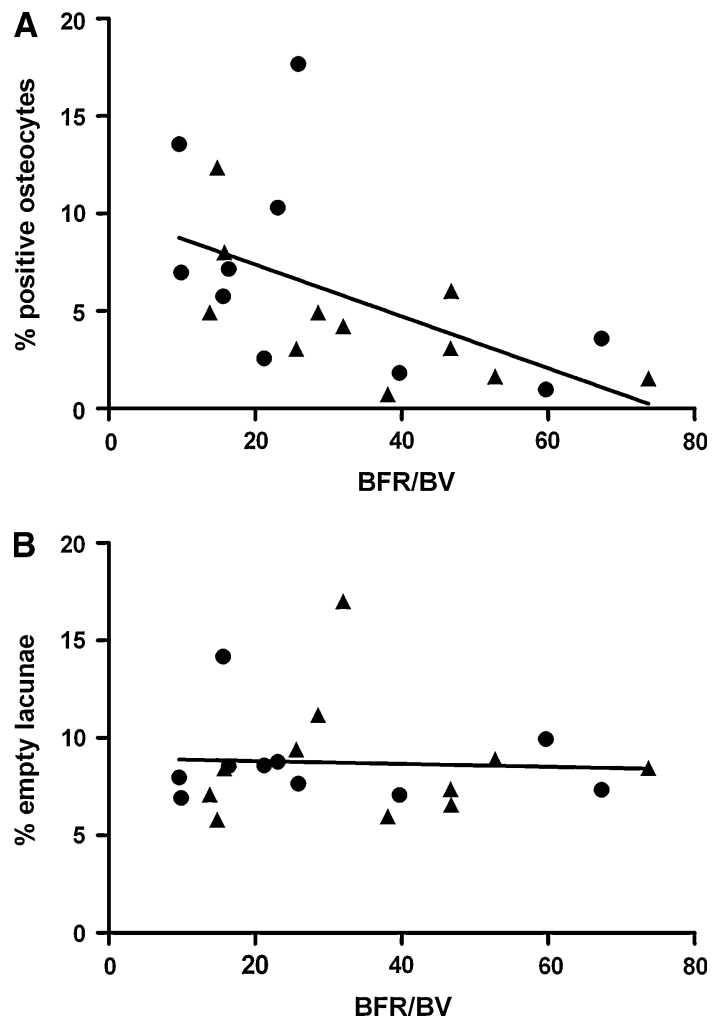

Fig. 3 Relationship between BFR/BV and osteocyte apoptosis at baseline in the placebo and raloxifene groups combined. (A) Association between BFR/BV and percent positive osteocytes. Correlation is significant for placebo and raloxifene groups combined $(P=0.001)$. (B) Association between BFR/BV and percentage empty lacunae. No significant correlation $(P=0.67)$. $\boldsymbol{O}$, placebo group; $\boldsymbol{\Lambda}$, raloxifene group
[24], although remnants of apoptotic cells, such as apoptotic bodies, might exist a little longer. Therefore, the cleaved caspase-3-positive osteocytes that were detected are cells that became apoptotic in the last 16 weeks of the treatment period. Moderate changes due to treatment with raloxifene are probably not detectable in such a short period. Empty lacunae remain in bone until bone remodeling will remove them, and this period is longer then the 16 weeks that an apoptotic osteocyte is detectable.

Changes in bone remodeling could have influenced the percentage of apoptotic osteocytes and empty lacunae that we found. The inverse correlation between bone remodeling indices and osteocyte apoptosis at baseline indicates that the level of osteocyte apoptosis is indeed associated with the level of bone remodeling. In our opinion, the explanation for this association is that with high bone remodeling the chance that apoptotic osteocytes are removed and replaced by new osteocytes is also high and, therefore, the percentage of detected apoptotic osteocytes is low. With low bone remodeling the chance that apoptotic osteocytes are removed and replaced by new osteocytes is also low and, therefore, the resulting percentage of observed apoptotic osteocytes is high. In this way, bone remodeling partly defines the percentage of apoptotic osteocytes, a mechanism which has already been suggested by Dunstan et al. [25]. This dependence of the level of osteocyte apoptosis on the level of bone remodeling complicates the detection of an effect of a treatment on osteocyte apoptosis if that treatment also has an effect on bone remodeling. In this study, there were no significant effects of raloxifene on bone remodeling parameters both in the placebo group and in the raloxifene

Table 3 Results of baseline and follow-up biopsies for histomorphometric indices and biochemical markers of bone remodeling

\begin{tabular}{llll}
\hline Parameter & Time point $\%$ change & Placebo $(n=11$, mean \pm SD $)$ & Raloxifene $^{\mathrm{a}}(n=15$, mean \pm SD $)$ \\
\hline OS/BS $(\%)$ & Baseline & $10.2 \pm 6.8$ & $9.9 \pm 5.4$ \\
& Follow-up & $7.5 \pm 3.2$ & $11.6 \pm 4.6$ \\
BFR/BV (\%/year) & Baseline & $28.8 \pm 20.3$ & $35.3 \pm 18.6$ \\
& Follow-up & $19.8 \pm 8.7$ & $23.3 \pm 12.6$ \\
ES/BS $(\%)$ & Baseline & $5.3 \pm 2.6$ & $6.3 \pm 2.9$ \\
& Follow-up & $5.3 \pm 2.4$ & $6.3 \pm 2.4$ \\
Ocl.N./B.Ar $\left(n / \mathrm{mm}^{2}\right)$ & Baseline & $0.6 \pm 0.3$ & $0.8 \pm 0.4$ \\
& Follow-up & $0.6 \pm 0.3$ & $0.6 \pm 0.3$ \\
BSAP $(\mu \mathrm{g} / \mathrm{L})$ & Baseline & $14.9 \pm 7.3$ & $14.4 \pm 4.6$ \\
& Follow-up & $12.2 \pm 5.6$ & $10.0 \pm 2.5^{\mathrm{b}}$ \\
Osteocalcin $(\mathrm{ng} / \mathrm{mL})$ & Baseline & $22.9 \pm 10.6$ & $25.5 \pm 9.1$ \\
CTX-1 $(\mu \mathrm{g} / \mathrm{mmol}$ creatinine) & Follow-up & $18.2 \pm 3.8$ & $16.1 \pm 3.1^{\mathrm{b}}$ \\
& Baseline & $283.9 \pm 267.0$ & $277.4 \pm 198.2$
\end{tabular}

${ }^{a}$ The $60 \mathrm{mg} /$ day and $120 \mathrm{mg} /$ day groups were combined

b Significantly different from baseline $(P<0.05)$ 
group; however, changes in individual women over the 2year treatment period could have influenced the level of osteocyte apoptosis found at follow-up. This could have obscured detection of a possible effect of raloxifene on osteocyte apoptosis.

The inverse correlation between osteocyte apoptosis and bone remodeling seems to be in contrast with the hypothesis put forward by several investigators $[5,26]$ that viable osteocytes inhibit bone remodeling and that lack of viable osteocytes, e.g., near sites of microdamage, attracts bone remodeling. We believe that our results do not contradict this hypothesis. Attraction of osteoclasts by dead osteocytes or by lack of osteocytes is probably a local process. If the occurrence of dead osteocytes and empty lacunae stimulates bone remodeling, the lacunae are more quickly removed and replaced with viable osteocytes.

The percent empty lacunae was not correlated to histomorphometric indices of bone remodeling. This suggests that the empty lacunae for a large part exist and increase in number in bone that is not participating in remodeling. In two studies, Qiu et al. [27, 28] have made the distinction between superficial bone and deep bone, i.e., bone at the surface of trabeculae and bone in the center of trabeculae. They postulated that deep bone is remodeled much more slowly than superficial bone. In this study, we did not make a distinction between superficial bone and deep bone, but it is possible that the increase in empty lacunae that we found primarily occurred in deep bone.

Biochemical markers of bone formation and resorption were not correlated with osteocyte apoptosis or with empty lacunae. Biochemical markers reflect bone remodeling in the whole skeleton, both in trabecular bone and in cortical bone. Correlations between such general markers and parameters measured locally in trabecular bone of the iliac crest are possibly more difficult to find.

The major limitation of our study is the small number of patients. The percentage of apoptotic osteocytes varied considerably between individuals, and this makes it difficult to find significant differences between such small groups. The variation was not caused by variation in the method, as shown by the low average standard deviation, indicating that the percentage of apoptotic osteocytes has to be an individual characteristic.

Detection of cleaved caspase-3, a key protease in the apoptotic process, is an established method for measuring apoptosis [29, 30]; and it has recently been used on bone tissue [31]. In the latter study, comparison between the TUNEL method and the cleaved caspase- 3 method showed no significant differences. We confirmed the specificity of the detection of cleaved caspase- 3 in sections of human ileum. In these sections, only the cells which are expected to be apoptotic [32] were stained by our method. Necrotic death of osteocytes might also occur in bone; however, we expect that necrotic cell death is low and not responsive to interventions such as estrogen, glucocorticoids, or mechanical loading. The average percentage apoptotic osteocytes we found was somewhat lower than what Tomkinson et al. [8] found in their study in young women who had received gonadotropin-releasing hormone analogue therapy. This could reflect differences in age and in treatment between the two studies.

The number of empty lacunae that we observed could be an overestimation caused by sectioning artefacts or the decalcification step in the immunohistochemistry method. We do not know to what extent this occurs, but this would be equal in all biopsies. Goldner-stained sections showed more sectioning artefacts, and therefore, empty lacunae in these sections were not counted. Inconsistency between the change in percent empty lacunae and empty lacunar density in the placebo group is probably also related to the small number of patients studied.

In this study, we did not find clear evidence that treatment with raloxifene influences osteocyte apoptosis. No change in the percentage of apoptotic osteocytes was detected, while the changes in the empty lacunae were inconclusive and at best indirect evidence that osteocyte apoptosis had been changed by raloxifene. Compared to estrogen, raloxifene treatment shows a similar reduction of vertebral fractures (but not nonvertebral fractures) [10, 33] but has less potent positive effects on bone quality assessed by BMD measurement and bone histomorphometry [34, 35]. It is conceivable that the weaker effect of raloxifene on bone is related to only a weak antiapoptotic effect or lack of antiapoptotic action. This is, however, difficult to measure in a study with such a small number of subjects. It would be of interest to compare the effects of different antiresorptive treatments on osteocyte apoptosis in larger clinical studies and to compare the mechanisms by which they exert their effect.

In conclusion, we did not find evidence for an effect of raloxifene treatment on osteocyte apoptosis in postmenopausal women, but small effects of raloxifene treatment on osteocyte apoptosis cannot be excluded. The percent of apoptotic osteocytes was dependent on the level of bone remodeling in an individual.

Acknowledgments The authors thank Ania Oleksik and Nicolette Pliester for their help with collection of the data. P. L. has received grants from Eli Lilly and Company, Merck \& Co., Aventis, and Wyeth and served as a consultant for Eli Lilly and Company, Merck \& Co., Wyeth, and Servier.

\section{References}

1. Burger EH, Klein-Nulend J (1999) Mechanotransduction in bone - role of the lacuno-canalicular network. FASEB J 13(suppl):S101-S112 
2. Ajubi NE, Klein-Nulend J, Nijweide PJ, Vrijheid-Lammers T, Alblas MJ, Burger EH (1996) Pulsating fluid flow increases prostaglandin production by cultured chicken osteocytes - a cytoskeleton-dependent process. Biochem Biophys Res Commun 225:62-68

3. MacIntyre I, Zaidi M, Alam AS, Datta HK, Moonga BS, Lidbury PS, Hecker M, Vane JR (1991) Osteoclastic inhibition: an action of nitric oxide not mediated by cyclic GMP. Proc Natl Acad Sci USA 88:2936-2940

4. Verborgt O, Gibson GJ, Schaffler MB (2000) Loss of osteocyte integrity in association with microdamage and bone remodeling after fatigue in vivo. J Bone Miner Res 15:60-67

5. Noble BS, Peet N, Stevens HY, Brabbs A, Mosley JR, Reilly GC, Reeve J, Skerry TM, Lanyon LE (2002) Mechanical loading: biphasic osteocyte survival and the targeting of osteoclasts for bone destruction in rat cortical bone. Am J Physiol Cell Physiol 284:C934-C943

6. Manolagas SC, Kousteni S, Jilka RL (2002) Sex steroids and bone. Recent Prog Horm Res 57:385-409

7. Stepan JJ, Alenfeld F, Boivin G, Feyen JH, Lakatos P (2003) Mechanisms of action of antiresorptive therapies of postmenopausal osteoporosis. Endocr Regul 37:225-238

8. Tomkinson A, Reeve J, Shaw RW, Noble BS (1997) The death of osteocytes via apoptosis accompanies estrogen withdrawal in human bone. J Clin Endocrinol Metab 82:3128-3135

9. Tomkinson A, Gevers EF, Wit JM, Reeve J, Noble BS (1998) The role of estrogen in the control of rat osteocyte apoptosis. J Bone Miner Res 13:1243-1250

10. Ettinger B, Black DM, Mitlak BH, Knickerbocker RK, Nickelsen T, Genant HK, Christiansen C, Delmas PD, Zanchetta JR, Stakkestad J, Gluer CC, Krueger K, Cohen FJ, Eckert S, Ensrud KE, Avioli LV, Lips P, Cummings SR (1999) Reduction of vertebral fracture risk in postmenopausal women with osteoporosis treated with raloxifene: results from a 3-year randomized clinical trial. Multiple Outcomes of Raloxifene Evaluation (MORE) Investigators. JAMA 282:637-645

11. Delmas PD, Ensrud KE, Adachi JD, Harper KD, Sarkar S, Gennari C, Reginster JY, Pols HA, Recker RR, Harris ST, Wu W, Genant HK, Black DM, Eastell R (2002) Efficacy of raloxifene on vertebral fracture risk reduction in postmenopausal women with osteoporosis: four-year results from a randomized clinical trial. J Clin Endocrinol Metab 87:3609-3617

12. Taranta A, Brama M, Teti A, De Luca V, Scandurra R, Spera G, Agnusdei D, Termine JD, Migliaccio S (2002) The selective estrogen receptor modulator raloxifene regulates osteoclast and osteoblast activity in vitro. Bone 30:368-376

13. Kousteni S, Han L, Chen JR, Almeida M, Plotkin LI, Bellido T, Manolagas SC (2003) Kinase-mediated regulation of common transcription factors accounts for the bone-protective effects of sex steroids. J Clin Invest 111:1651-1664

14. Theuns HM, Bekker H, Fokkenrood H, Offerman E (1993) Methyl-methacrylate embedding of undecalcified rat bone and simultaneous staining for alkaline and tartrate resistant acid phosphatase. Bone 14:545-550

15. Oleksik A, Ott SM, Vedi S, Bravenboer N, Compston J, Lips P (2000) Bone structure in patients with low bone mineral density with or without vertebral fractures. J Bone Miner Res 15:13681375

16. Ott SM, Oleksik A, Lu Y, Harper K, Lips P (2002) Bone histomorphometric and biochemical marker results of a 2-year placebo-controlled trial of raloxifene in postmenopausal women. $\mathrm{J}$ Bone Miner Res 17:341-348

17. Parfitt AM, Drezner MK, Glorieux FH, Kanis JA, Malluche H, Meunier PJ, Ott SM, Recker RR (1987) Bone histomorphometry: standardization of nomenclature, symbols, and units. Report of the ASBMR Histomorphometry Nomenclature Committee. J Bone Miner Res 2:595-610

18. Rauch F (2006) Watching bone cells at work: what we can see from bone biopsies. Pediatr Nephrol 21:457-462

19. Olivier S, Fillet M, Malaise M, Piette J, Bours V, Merville MP, Franchimont N (2005) Sodium nitroprusside-induced osteoblast apoptosis is mediated by long chain ceramide and is decreased by raloxifene. Biochem Pharmacol 69:891-901

20. Plotkin LI, Aguirre JI, Kousteni S, Manolagas SC, Bellido T (2005) Bisphosphonates and estrogens inhibit osteocyte apoptosis via distinct molecular mechanisms downstream of extracellular signal-regulated kinase activation. J Biol Chem 280:7317-7325

21. Gu G, Hentunen TA, Nars M, Harkonen PL, Vaananen HK (2005) Estrogen protects primary osteocytes against glucocorticoid-induced apoptosis. Apoptosis 10:583-595

22. Plotkin LI, Weinstein RS, Parfitt AM, Roberson PK, Manolagas SC, Bellido T (1999) Prevention of osteocyte and osteoblast apoptosis by bisphosphonates and calcitonin. J Clin Invest 104:1363-1374

23. Gohel A, McCarthy MB, Gronowicz G (1999) Estrogen prevents glucocorticoid-induced apoptosis in osteoblasts in vivo and in vitro. Endocrinology 140:5339-5347

24. Kenzora JE, Steele RE, Yosipovitch ZH, Glimcher MJ (1978) Experimental osteonecrosis of the femoral head in adult rabbits. Clin Orthop Relat Res 130:8-46

25. Dunstan CR, Somers NM, Evans RA (1993) Osteocyte death and hip fracture. Calcif Tissue Int 53(suppl 1):S113-S116

26. Gu G, Mulari M, Peng Z, Hentunen TA, Vaananen HK (2005) Death of osteocytes turns off the inhibition of osteoclasts and triggers local bone resorption. Biochem Biophys Res Commun 335:1095-1101

27. Qiu S, Rao DS, Palnitkar S, Parfitt AM (2002) Age and distance from the surface but not menopause reduce osteocyte density in human cancellous bone. Bone 31:313-318

28. Qiu S, Rao DS, Palnitkar S, Parfitt AM (2002) Relationships between osteocyte density and bone formation rate in human cancellous bone. Bone 31:709-711

29. Duan WR, Garner DS, Williams SD, Funckes-Shippy CL, Spath IS, Blomme EA (2003) Comparison of immunohistochemistry for activated caspase- 3 and cleaved cytokeratin 18 with the TUNEL method for quantification of apoptosis in histological sections of PC-3 subcutaneous xenografts. J Pathol 199:221-228

30. Dukers DF, Oudejans JJ, Vos W, ten Berge RL, Meijer CJ (2002) Apoptosis in B-cell lymphomas and reactive lymphoid tissues always involves activation of caspase 3 as determined by a new in situ detection method. J Pathol 196:307-315

31. Basso N, Heersche JN (2006) Effects of hind limb unloading and reloading on nitric oxide synthase expression and apoptosis of osteocytes and chondrocytes. Bone 39:807-814

32. Grossmann J, Walther K, Artinger M, Rummele P, Woenckhaus M, Scholmerich J (2002) Induction of apoptosis before shedding of human intestinal epithelial cells. Am J Gastroenterol 97:14211428

33. Marcus R, Wong M, Heath H III, Stock JL (2002) Antiresorptive treatment of postmenopausal osteoporosis: comparison of study designs and outcomes in large clinical trials with fracture as an endpoint. Endocr Rev 23:16-37

34. Prestwood KM, Gunness M, Muchmore DB, Lu Y, Wong M, Raisz LG (2000) A comparison of the effects of raloxifene and estrogen on bone in postmenopausal women. J Clin Endocrinol Metab 85:2197-2202

35. Weinstein RS, Parfitt AM, Marcus R, Greenwald M, Crans G, Muchmore DB (2003) Effects of raloxifene, hormone replacement therapy, and placebo on bone turnover in postmenopausal women. Osteoporos Int 14:814-822 